\title{
Cluster-additive functions on stable translation quivers
}

\author{
Claus Michael Ringel
}

Received: 8 May 2011 / Accepted: 19 January 2012 / Published online: 14 February 2012

(C) Springer Science+Business Media, LLC 2012

\begin{abstract}
Additive functions on translation quivers have played an important role in the representation theory of finite-dimensional algebras, the most prominent ones are the hammock functions introduced by S. Brenner. When dealing with cluster categories (and cluster-tilted algebras), one should look at a corresponding class of functions defined on stable translation quivers, namely the cluster-additive ones. We conjecture that the cluster-additive functions on a stable translation quiver of Dynkin type $\mathbb{A}_{n}, \mathbb{D}_{n}, \mathbb{E}_{6}, \mathbb{E}_{7}, \mathbb{E}_{8}$ are non-negative linear combinations of cluster-hammock functions (with index set a tilting set). The present paper provides a first study of cluster-additive functions and gives a proof of the conjecture in the case $\mathbb{A}_{n}$.
\end{abstract}

Keywords Translation quiver - Additive function - Cluster-additive function · Hammocks · Cluster-hammocks · Dynkin quiver · Cluster category · Cluster-tilted algebra

A translation quiver is of the form $\Gamma=\left(\Gamma_{0}, \Gamma_{1}, \tau\right)$, where $\left(\Gamma_{0}, \Gamma_{1}\right)$ is a locally finite quiver say with $m_{x y}$ arrows $x \rightarrow y$, and $\tau:\left(\Gamma_{0} \backslash \Gamma_{0}^{p}\right) \rightarrow \Gamma_{0}$ is an injective function defined on the complement of a subset $\Gamma_{0}^{p} \subseteq \Gamma_{0}$, such that for any pair of vertices $y, z \in \Gamma_{0}$, with $z \notin \Gamma_{0}^{p}$ one has $m_{\tau z, y}=m_{y, z}$. The vertices in $\Gamma_{0}^{p}$ are said to be the projective vertices, those not in the image of $\tau$ the injective vertices. If there are neither projective nor injective vertices, then $\Gamma$ is said to be stable. A typical example of a translation quiver is the Auslander-Reiten quiver of a finite-dimensional $k$ algebra $A$, where $k$ is an algebraically closed field. Such an Auslander-Reiten quiver

C.M. Ringel ( $\square)$

Fakultät für Mathematik, Universität Bielefeld, P.O. Box 100 131, 33501 Bielefeld, Germany

e-mail: ringel@math.uni-bielefeld.de

C.M. Ringel

King Abdulaziz University, P.O. Box 80200, Jeddah, Saudi Arabia 
is equipped with an additive function on the set of vertices with values in the set of positive integers, its value at a vertex $x$ is the length of the corresponding $A$-module. Here, a function $f: \Gamma_{0} \rightarrow \mathbb{Z}$ is said to be additive provided

$$
f(z)+f(\tau z)=\sum_{y \in \Gamma_{0}} m_{y z} f(y), \quad \text { for all } z \in \Gamma_{0} \backslash \Gamma_{0}^{p} .
$$

The importance of dealing with additive functions on translation quivers is wellknown since a long time, of particular relevance have been the hammock functions introduced by Brenner [3], see also [14]; hammock functions for vertices of the translation quivers of the form $\Gamma=\mathbb{Z} \Delta$ with $\Delta$ a Dynkin quiver have been displayed already by Gabriel [9] in 1980.

The present note is concerned with combinatorial features of cluster categories (introduced by Buan, Marsh, Reineke, Reiten, Todorov [4], in the special case $\mathbb{A}_{n}$ also by Caldero, Chapoton, Schiffler [7]) and cluster-tilted algebras (introduced by Buan, Marsh, Reiten [5]), and for simplicity we again will assume that we work over an algebraically closed field. The cluster categories are triangulated categories with Auslander-Reiten triangles, thus we may consider the corresponding AuslanderReiten quivers: these are now stable translation quivers. Thus, let $\Gamma$ be a stable translation quiver. Instead of looking at additive functions on $\Gamma$, we now will be interested in what we call cluster-additive functions.

We use the following notation: Any integer $z$ can be written as $z=z^{+}-z^{-}$with non-negative integers $z^{+}, z^{-}$such that $z^{+} z^{-}=0$ (thus $z^{+}=\max \{z, 0\}$ and $z^{-}=$ $\max \{-z, 0\})$. A function $f: \Gamma_{0} \rightarrow \mathbb{Z}$ is said to be cluster-additive on $\Gamma$ provided

$$
f(z)+f(\tau z)=\sum_{y \in \Gamma_{0}} m_{y z} f(y)^{+}, \quad \text { for all } z \in \Gamma_{0} .
$$

Linear combinations of cluster-additive functions usually are not cluster-additive. Theorems 1 and 2 deal with this topic. Here, we assume that we deal with a stable translation quiver $\Gamma$ such that any vertex is starting point of an arrow (we call such a stable translation quiver proper; in the terminology of Riedtmann [13], it means that we assume that no component of $\Gamma$ has tree class $\mathbb{A}_{1}$ ). Theorem 1 provides a criterion for sums of cluster-additive functions to be cluster-additive again: If $f, g$ are clusteradditive functions on $\Gamma$, then $f+g$ is cluster-additive if and only if $f(x) g(x) \geq 0$ for all vertices $x$ (in this case, we say that $f$ and $g$ are compatible). Theorem 2 shows that the difference $f-g$ of cluster-additive functions $f, g$ is cluster-additive if and only if $g(x)^{+} \leq f(x)^{+}$and $g(x)^{-} \leq f(x)^{-}$for all vertices $x$ (if this is the case we write $g \leq f)$.

The remaining parts of the paper deals with translation quivers related to those of the form $\mathbb{Z} \Delta$ where $\Delta$ is assumed to be a finite directed quiver, or often even a Dynkin quiver. Recall that any locally finite directed quiver $\Delta$ gives rise to a stable translation quiver $\mathbb{Z} \Delta$ with vertex set $\Delta \times \mathbb{Z}$, with arrows $(\alpha, i):(\xi, i) \rightarrow(\eta, i)$ and $\left(\alpha^{*}, i\right):(\eta, i) \rightarrow(\xi, i+1)$ for any arrow $\alpha: \xi \rightarrow \eta$ in $\Delta$ and with translation $(\xi, i) \mapsto$ $(\xi, i-1)$. Theorem 3 asserts that $a$ cluster-additive function on $\mathbb{Z} \Delta$ with $\Delta$ a finite directed quiver is uniquely determined by its values on a section and that these values are arbitrary integers. Thus, if $\Delta$ has $n$ vertices, we may identify in this way the set 
of cluster-additive functions on $\Gamma$ with the set $\mathbb{Z}^{n}$; but note that this is just a settheoretical bijection!

Our main interest lies in the translation quivers $\mathbb{Z} \Delta$ where $\Delta$ is a Dynkin-quiver, thus the underlying graph is one of the simply laced Dynkin diagrams $\mathbb{A}_{n}, \mathbb{D}_{n}, \mathbb{E}_{6}$, $\mathbb{E}_{7}$, or $\mathbb{E}_{8}$. Theorem 4 asserts that for $\Delta$ of type $\mathbb{A}_{n}$, any cluster-additive function on $\mathbb{Z} \Delta$ is a non-negative linear combination of cluster-hammock functions (they are introduced in Sect. 5). We conjecture that the same assertion holds for all Dynkin cases. This would be an analog of an old theorem of Butler [6] which asserts that for a representation-finite algebra $A$, the additive functions on the Auslander-Reiten quiver of $A$ are the linear combinations of the hammock functions.

Cluster-additive functions arise naturally in the context of cluster categories and cluster-tilted algebras (see Sect. 10), thus one may be tempted to focus the attention to cluster-additive functions on stable translation quivers $\Gamma$ such as the AuslanderReiten quiver of a cluster category, a typical example is $\mathbb{Z} \Delta / F$ where $\Delta$ is a Dynkin quiver and $F=\tau^{-1}[1]$. It may come as a surprise that instead of looking at $\mathbb{Z} \Delta / F$, we prefer to consider cluster-additive functions on its cover $\mathbb{Z} \Delta$. After all, every cluster-additive function on $\mathbb{Z} \Delta / F$ lifts to a cluster-additive function on $\mathbb{Z} \Delta$, thus we deal with a setting which on a first sight appears to be more general. But we conjecture that all the cluster-additive functions on $\mathbb{Z} \Delta$ actually are $F$-invariant, so that we would get the shift $F$ for free.

The experienced reader will observe that the cluster-additive functions exhibit a lot of typical features known in cluster theory (as started by Fomin and Zelevinsky [8] and developed further by a large number of mathematicians): that negative numbers arise only seldom, that they have to be ignored in some calculations, that there is a playing field which concerns only non-negative numbers, and if the ball leaves the field, it is bounced back immediately ....

One of the referees of the paper suggested to mention here the "frieze functions" introduced by Assem, Reutenauer, Smith [1], since they also assign integers to vertices in Auslander-Reiten quivers; the frieze functions are related to the numerators of cluster variables, whereas the cluster additive functions are related to the denominators.

\section{Preliminaries}

Let $\Gamma$ be a stable translation quiver. We compare additivity and cluster-additivity and look for the image of a cluster-additive function.

A special cases should be discussed beforehand, namely the stable translation quivers $\Gamma$ without arrows. In this case, a function $f: \Gamma_{0} \rightarrow \mathbb{Z}$ is cluster-additive if and only if $f$ is additive, if and only if $f(\tau z)=-f(z)$ for all vertices $z$. If we consider the $\tau$-orbit of the vertex $z$, then two possibilities have to be distinguished: either the $\tau$-orbit has an odd number of elements, then we must have $f(z)=0$ for any cluster-additive function on $\Gamma$, or else the $\tau$-orbit is infinite or has an even number of elements, then $f(z)$ is an arbitrary integer, and determines the values of $f$ on the complete $\tau$-orbit if we label the vertices as $x_{i}$ with $i \in \mathbb{Z}$ such that $\tau x_{i}=x_{i-1}$, the cluster-additive functions are the functions of the form $f\left(x_{i}\right)=(-1)^{i} a$, where $a$ is a 
fixed integer. Note that this implies that in this special case, the set of cluster-additive functions is closed under (pointwise) addition and subtraction.

Let us call a stable translation quiver $\Gamma$ proper, provided any vertex is starting point of at least one arrow (in the terminology of Riedtmann [13], this can be formulated as follows: no component of $\Gamma$ is of tree class $\mathbb{A}_{1}$ ). Observe that a connected stable translation quiver with at least one arrow is always proper! The stable translation quivers we are interested in will always be proper. We start with some properties of functions $f: \Gamma_{0} \rightarrow \mathbb{Z}$. The first property is obvious:

(1) A function $f$ on $\Gamma_{0}$ with values in $\mathbb{N}_{0}$ is cluster-additive if and only if it is additive.

(2) If $\Gamma$ is a proper translation quiver, then any function $f: \Gamma_{0} \rightarrow \mathbb{Z}$ which is both additive and cluster-additive takes values in $\mathbb{N}_{0}$.

Proof Let $y_{0} \in \Gamma_{0}$. Since $\Gamma$ is proper, any vertex is the starting point of an arrow, thus there is an arrow $y_{0} \rightarrow z$. Now

$$
f(z)+f(\tau z)=\sum_{y} m_{y z} f(y)^{+}=\sum_{y} m_{y z} f(y)
$$

implies that $\sum_{y} m_{y z}\left(f(y)^{+}-f(y)\right)=0$. However we have $f(y)^{+}-f(y) \geq 0$ for all $y$. This shows that for $m_{y z} \neq 0$ we must have $f(y)^{+}=f(y)$. Since $m_{y_{0}, z} \neq 0$, we see that $f\left(y_{0}\right)^{+}=f\left(y_{0}\right)$. Thus $f\left(y_{0}\right) \geq 0$.

(3) Let $f$ be cluster-additive. Let $f(z)<0$. Then $f(\tau z) \geq-f(z)>0$.

Proof By definition, $f(\tau z)+f(z)$ is a sum of non-negative numbers, thus nonnegative.

This shows:

(4) Any cluster-additive function with only non-positive values is the zero function.

(5) Let $\Gamma=\mathbb{Z} \Delta$ with $\Delta$ of Dynkin type. Any cluster-additive function on $\Gamma$ with only non-negative values is the zero function.

Proof Let $f$ be cluster-additive on $\Gamma$ with only non-negative values. Then $f$ is additive, but according to [11] any additive function on $\Gamma$ with only non-negative values is the zero function.

It follows from (1), (3) and (5) that there are many stable translation quivers without non-zero cluster-additive functions. For example, if $\Delta$ is a Dynkin quiver, then the only cluster-additive function $f$ on $\Gamma=\mathbb{Z} \Delta / \tau$ is the zero function. Namely, (3) asserts that $f$ only takes non-negative values, thus $f$ is additive by (1). This means that $f$ is an additive function on $\mathbb{Z} \Delta$ with non-negative values. According to (5) this implies that $f$ is the zero function. 


\section{Sums of cluster-additive functions}

The sum of two cluster-additive functions usually will not be cluster-additive, a typical example is the following:

Example Let $\Gamma=\mathbb{Z} A_{2}$.

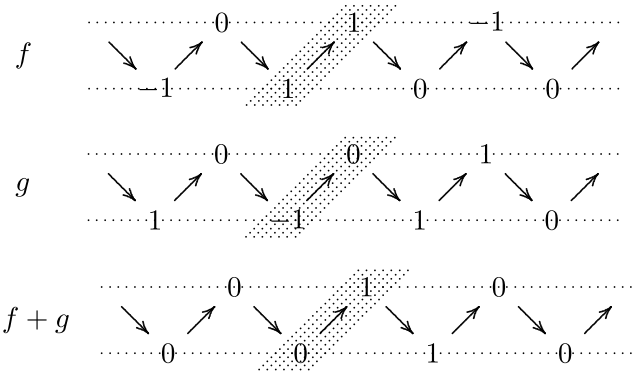

As we have mentioned, if $\Gamma$ is a stable translation quiver of tree class $\mathbb{A}_{1}$, then the set of cluster-additive functions on $\Gamma$ is closed under addition. Thus, let us assume now that $\Gamma$ is proper.

Two cluster-additive functions $f, g$ on $\Gamma$ are said to be compatible provided $f(x) g(x) \geq 0$ for all vertices $x$. Compatibility can be characterized in many different ways (the proof is obvious):

Lemma Let $f_{1}, \ldots, f_{n}$ be cluster-additive functions on $\Gamma$. The following conditions are equivalent:

(i) $f_{1}, \ldots, f_{n}$ are pairwise compatible.

(ii) If $f_{i}(x)<0$ for some index $i$ and some vertex $x$, then $f_{j}(x) \leq 0$ for $1 \leq j \leq n$.

(iii) If $f_{i}(x)>0$ for some index $i$ and some vertex $x$, then $f_{j}(x) \geq 0$ for $1 \leq j \leq n$.

(iv) Given a pair $i \neq j$, there is no vertex $x$ with $f_{i}(x)<0$ and $f_{j}(x)>0$.

Theorem 1 Let $f_{1}, \ldots, f_{n}$ be cluster-additive functions on a proper stable translation quiver $\Gamma$. Then $\sum f_{i}$ is cluster-additive if and only if the functions are pairwise compatible.

Before we start with the proof, let us isolate a decisive property of the operator $z \mapsto z^{+}$.

Lemma Let $a_{1}, \ldots, a_{n}$ be integers. Then

(a) $\left(\sum_{i} a_{i}\right)^{+} \leq \sum_{i} a_{i}^{+}$.

(b) Equality holds if and only if either all $a_{i}$ are non-negative or all are non-positive. 
Proof Let $a_{i} \geq 0$ for $1 \leq i \leq m$ and $a_{i} \leq 0$ for $m+1 \leq i \leq n$, let $a=\sum_{i=1}^{n} a_{i}$. Then $\sum_{i=1}^{n} a_{i}^{+}=\sum_{i=1}^{m} a_{i} \geq \sum_{i=1}^{n} a_{i}=a$ and therefore $\sum_{i=1}^{n} a_{i}^{+} \geq a^{+}$. If we have equality, and $a \geq 0$, then $0=a-\sum_{i=1}^{n} a_{i}^{+}=\sum_{i=m+1}^{n} a_{i}$ shows that $a_{i}=0$, for $m+1 \leq i \leq n$ (since these $a_{i}$ are non-positive). Thus all the $a_{i}$ are non-negative in this case.

If $a \leq 0$, then $\sum_{i=1}^{m} a_{i}=0$ shows that these $a_{i}=0$, since all $a_{i}$ are non-negative for $1 \leq i \leq m$. In this case, all $a_{i}$ are non-positive.

Also the converse holds: If all $a_{i}$ are non-negative, then $\sum_{i=1}^{n} a_{i}^{+}=\sum_{i=1}^{n} a_{i}=$ $\left(\sum_{i=1}^{n} a_{i}\right)^{+}$. If all $a_{i}$ are non-positive, then also $\sum_{i=1}^{n} a_{i}$ is non-positive, and $\sum_{i=1}^{n} a_{i}^{+}=0=\left(\sum_{i=1}^{n} a_{i}\right)^{+}$.

Proof of Theorem 1 Since we assume that $\Gamma$ is proper, we know that for any vertex $y$ in $\Gamma$, there is a vertex $z$ with $m_{y z} \neq 0$.

First let us assume that $f_{1}, \ldots, f_{a}$ are pairwise compatible and let $f=\sum_{i} f_{i}$. We claim that for all vertices $y$ of $\Gamma$

$$
f(y)^{+}=\sum_{i} f_{i}(y)^{+}
$$

Let $\mathcal{T}$ be the set of vertices $x \in \Gamma_{0}$ such that $f_{i}(x)<0$ for at least one $i$. If $y \in \mathcal{T}$, then $f_{i}(y) \leq 0$ for all $1 \leq i \leq n$, since we deal with pairwise compatible functions. It follows that $f(y)=\sum_{i} f_{i}(y)<0$ and therefore $f(y)^{+}=0$. But also $f_{i}(y)^{+}=0$ for all $i$, this yields $(*)$ in case $y \in \mathcal{T}$.

Now assume $y \notin \mathcal{T}$. Then $f_{i}(y) \geq 0$ for all $i$, thus $f(y)=\sum_{i} f_{i}(y) \geq 0$, therefore

$$
f(y)^{+}=f(y)=\sum_{i} f_{i}(y)=\sum_{i} f_{i}(y)^{+},
$$

and we see that $(*)$ is satisfied also in this case.

Now consider some vertex $z$.

$$
\begin{aligned}
f(\tau z)+f(z) & =\sum_{i} f_{i}(\tau z)+\sum_{i} f_{i}(z)=\sum_{i}\left(f_{i}(\tau z)+f_{i}(z)\right) \\
& =\sum_{i}\left(\sum_{y} m_{y z} f_{i}(y)^{+}\right) \\
& =\sum_{y} m_{y z} \sum_{i} f_{i}(y)^{+} \\
& =\sum_{y} m_{y z} f(y)^{+},
\end{aligned}
$$

where we use that all the functions $f_{i}$ are cluster additive as well as the equality $(*)$ for all $y$. This shows that $f$ is cluster additive.

Now let us assume that $f=\sum f_{i}$ is cluster additive. Let $z$ be a vertex of $\Gamma$. Then, as above, we have

$$
f(\tau z)+f(z)=\sum_{i} f_{i}(\tau z)+\sum_{i} f_{i}(z)=\sum_{i}\left(f_{i}(\tau z)+f_{i}(z)\right)
$$




$$
\begin{aligned}
& =\sum_{i}\left(\sum_{y} m_{y z} f_{i}(y)^{+}\right) \\
& =\sum_{y} m_{y z} \sum_{i} f_{i}(y)^{+},
\end{aligned}
$$

thus

$$
\begin{aligned}
0 & =f(\tau z)+f(z)-\sum_{i} m_{y z} f(y)^{+} \\
& =\sum_{y} m_{y z} \sum_{i} f_{i}(y)^{+}-\sum_{y} m_{y z} f(y)^{+} \\
& =\sum_{y} m_{y z}\left(\sum_{i} f_{i}(y)^{+}-f(y)^{+}\right) .
\end{aligned}
$$

According to assertion (a) of the Lemma, all the brackets in the last line are nonnegative, thus all the summands $m_{y z}\left(\sum_{i} f_{i}(y)^{+}-f(y)^{+}\right)$are non-negative. Since their sum is zero, all these summands are zero.

It follows that for any $y$ we have

$$
\sum_{i} f_{i}(y)^{+}=f(y)^{+}
$$

(since there is $z$ with $m_{y z} \neq 0$ ). According to the assertion (b) of the Lemma, we conclude that all the values $f_{i}(y)$ for $1 \leq i \leq n$ are non-negative or all are nonpositive. But this means that the functions $f_{1}, \ldots, f_{n}$ are compatible.

\section{Subtraction}

Let us introduce the following partial ordering on the set of cluster additive functions on $\Gamma$. If $f, g$ are cluster additive functions on $\Gamma$, we write $g \leq f$ provided $g(x)^{+} \leq$ $f(x)^{+}$as well as $g(x)^{-} \leq f(x)^{-}$for all vertices $x$ of $\Gamma$. (Note that $g \leq f$ implies that $|g(x)| \leq|f(x)|$ for all vertices $x$.)

Theorem 2 Let $f, g$ be cluster additive functions on the proper stable translation quiver $\Gamma$. Then $f-g$ is cluster additive if and only if $g \leq f$.

Proof First, let us assume that $g \leq f$. We claim that

$$
(f-g)(x)^{+}=f(x)^{+}-g(x)^{+}
$$

for all vertices $x$. Namely, if $g(x)>0$, then $g(x)=g(x)^{+} \leq f(x)^{+}$, and therefore $f(x)=f(x)^{+}$, thus $g(x) \leq f(x)$ and therefore $(f-g)(x)=f(x)-g(x) \geq 0$, thus

$$
(f-g)(x)^{+}=(f-g)(x)=f(x)-g(x)=f(x)^{+}-g(x)^{+} .
$$


Also, if $g(x)<0$, then $g(x)^{+}=0$, and $0<-g(x)=g(x)^{-} \leq f(x)^{-}$, thus $f(x)^{+}=$ 0. Also, $f(x)^{-}=-f(x)$ and therefore $g(x) \geq f(x)$, thus $(f-g)(x)=f(x)-$ $g(x) \leq 0$. It follows that

$$
(f-g)(x)^{+}=0=f(x)^{+}-g(x)^{+} .
$$

Finally, if $g(x)=0$, then also $g(x)^{+}=0$ and

$$
(f-g)(x)^{+}=f(x)^{+}=f(x)^{+}-g(x)^{+} .
$$

Let $z$ be a vertex of $\Gamma$, then

$$
\begin{aligned}
(f-g)(\tau z)+(f-g)(z) & =f(\tau z)-f(z)+g(\tau z)-g(z) \\
& =\sum_{y} m_{y z} f(y)^{+}-\sum_{y} m_{y z} g(y)^{+} \\
& =\sum_{y} m_{y z}(f-g)(y)^{+} .
\end{aligned}
$$

This shows that $f-g$ is cluster additive.

Conversely, assume that $f-g$ is cluster additive. Since the sum $f=(f-g)+g$ of the cluster additive functions $f-g$ and $g$ is cluster additive, we know by Theorem 1 that $f-g$ and $g$ are compatible functions, thus $(f-g)(x) g(x) \geq 0$ for all vertices $x$, thus $f(x) g(x) \geq g(x) g(x)$ for all $x$. If $g(x)>0$, then this implies that $f(x) \geq g(x)>0$, thus $f(x)^{+} \geq g(x)^{+}$and $f(x)^{-}=g(x)^{-}$. If $g(x)<0$, then $f(x) \leq g(x)<0$, therefore $g(x)^{-}=-g(x) \leq-f(x)=f(x)^{-}$and $g(x)^{+}=0=$ $f(x)^{+}$. Of course, if $g(x)=0$, then $g(x)^{+}=0 \leq f(x)^{+}$and $g(x)^{-}=0 \leq f(x)^{-}$. This shows that $g \leq f$.

\section{The restriction of cluster additive functions to a section}

We consider now cluster additive functions on a translation quiver $\Gamma=\mathbb{Z} \Delta$, where $\Delta$ is a (usually finite) directed quiver. The subset $\Delta_{0} \times\{0\}$ is a section of $\Gamma$ (by definition, the sections of $\mathbb{Z} \Delta$ are the subsets $\eta\left(\Delta_{0}^{\prime} \times\{0\}\right)$, where $\eta: \mathbb{Z} \Delta^{\prime} \rightarrow \mathbb{Z} \Delta$ is an isomorphism of translation quivers).

Theorem 3 Let $\Delta$ be a finite directed quiver. Any function $f: \Delta_{0} \times\{0\} \rightarrow \mathbb{Z}$ can be extended uniquely to a cluster additive function on $\mathbb{Z} \Delta$.

This may be reformulated as follows:

Corollary 1 The restriction furnishes a bijection between the set of cluster additive functions on $\mathbb{Z} \Delta$ and the functions $f: \Delta_{0} \times\{0\} \rightarrow \mathbb{Z}$.

Proof of Theorem 3 Let $\xi$ be a source in $\Delta$. Then $f$ is defined for $(\xi, 0)$ and all its direct successors, thus we use the defining property of a cluster additive function in order to define $f(\xi, 1)$. Inductively we define in this way $f(\eta, j)$ for all vertices $\eta$ of $\Delta$ and all $j>0$. The dual procedure yields the values $f(\eta, j)$ for $j<0$. 
Remark 1 Note that we need here that $\Delta$ is finite. For example, if $\Delta$ is the linearly ordered quiver of type $\mathbb{A}_{\infty}^{\infty}$, then any function $f: \mathbb{Z} \Delta \rightarrow \mathbb{N}_{0}$ which is constant on the sections $\Delta \times\{i\}$, for all $i \in \mathbb{Z}$, is additive, thus cluster additive and of course not determined by the value taken on one of these sections.

We also may look at $\Gamma=\mathbb{Z} \Delta$ with $\Delta$ a locally finite (but not necessarily finite) directed quiver. A section $S$ of $\Gamma$ may be said to be generating provided we obtain all vertices from $S$ of $\Gamma$ using reflections (see [2]) at sinks and at sources. If $\Delta$ a finite, then any section is generating, but in general not. If $\Gamma=\mathbb{Z} A_{\infty}^{\infty}$, then a section $S$ is generating if and only if no arrow in $S$ belongs to an infinite path. The corollary can be generalized as follows: Let $S$ be a generating section. Then the restriction function $f \mapsto f \mid S$ is bijective.

Remark 2 The extension of a function $f: \Delta_{0} \times\{i\} \rightarrow \mathbb{Z}$ to a cluster additive function on $\Gamma$ can be achieved by using what one may call cluster reflections. Given a locally finite quiver $\Delta$ and a vertex $x$ of $\Delta$, which is a sink or a source, then the cluster reflection $\sigma_{x}$ maps any function $f: \Delta_{0} \rightarrow \mathbb{Z}$ to the function $\sigma_{x} f$ with $\left(\sigma_{x} f\right)(y)=f(y)$ for $y \neq x$ and $\left(\sigma_{x} f\right)(x)=-f(x)+\sum_{y} m_{x y} f(y)^{+}$and $\sigma_{x} f$ should be considered as a function on $\left(\sigma_{x} \Delta\right)_{0}$, where $\sigma_{x} \Delta$ is obtained from $\Delta$ by changing the orientation of all the arrows involving $x$ (thus replacing a source by a sink and vice versa). Starting with a source $x$ of $\Delta=\Delta \times\{i\}$, then we may identify $\sigma_{x} \Delta$ with the section obtained by deleting $x$ and adding $\tau^{-1} x$; given a function $f: \Delta \rightarrow \mathbb{Z}$, and looking for its cluster additive extension, then we have to use $\sigma_{x} f$ on the section $\sigma_{x} \Delta$.

Altogether we see that the restrictions of a cluster additive function on $\Gamma$ to the various sections of $\Gamma$ are obtained from each other by a sequence of cluster reflections.

\section{Cluster-hammock functions}

Here we introduce some basic cluster additive functions. As before, we consider a translation quiver $\Gamma=\mathbb{Z} \Delta$, where $\Delta$ is a finite directed quiver, but later we will assume that $\Delta$ is a Dynkin quiver.

Recall the definition of the left hammock function $h_{p}^{\prime}$ for a vertex $p$ of $\Gamma$ (and that left hammock functions with finite support are called hammock functions). First, $h_{p}^{\prime}(p)=1$. Second, if $z$ is not a successor of $p$, then $h_{p}^{\prime}(z)=0$. Third, assume that $h_{p}^{\prime}(y)$ is defined for all proper predecessors $y$ of $z$; if there is an arrow $y \rightarrow z$ with $h_{p}^{\prime}(y)>0$, then

$$
h_{p}^{\prime}(z)=-h_{p}^{\prime}(\tau z)+\sum_{y} m_{y z} h_{p}^{\prime}(y)
$$

otherwise $h_{p}^{\prime}(z)=0$.

It is well-known that all the values $h_{p}^{\prime}(z)$ are non-negative; the support of $h_{p}^{\prime}$ will be denoted by $H_{p}$. If $\Delta$ is a Dynkin quiver (thus of type $\mathbb{A}_{n}, \mathbb{D}_{n}, \mathbb{E}_{6}, \mathbb{E}_{7}$, or $\mathbb{E}_{8}$ ), then $H_{p}$ is finite and there is a unique vertex $v p$ with $h_{p}^{\prime}(v p) \neq 0$ such that any vertex $y$ 
with $h_{p}^{\prime}(y) \neq 0$ is a predecessor of $v p$; the map $v: \Gamma_{0} \rightarrow \Gamma_{0}$ is called the Nakayama shift (see [9], where also typical hammock functions are displayed; but note that in contrast to the definition given in this paper, but also in [3] and [14], Gabriel extends the function $h_{p}^{\prime} \mid H_{p}$ to an additive function on all of $\mathbb{Z} \Delta$ ). The shift $\nu \tau^{-1}$ is usually denoted by [1], the shift $\nu \tau^{-2}$ by $F$.

We insert here that $\mathbb{Z} \Delta$ for $\Delta$ a Dynkin quiver may be interpreted as the Auslander-Reiten quiver of the derived category $D^{b}(\bmod A)$, where $A$ is the path algebra of the opposite quiver of $\Delta$, see [10]. Given an indecomposable $A$-module $X$, we denote by $[X]$ the corresponding vertex in $\mathbb{Z} \Delta$. In this interpretation, [1] corresponds to the shift functor of the derived category and $F$ to the functor [1] $\tau^{-1}$ (also denoted by $F$ ) which is used in order to define the corresponding cluster category, see [4].

If $\Delta$ is connected and not one of these Dynkin quivers, then the support $H_{p}$ of $h_{p}^{\prime}$ is infinite, for any vertex $p$ of $\mathbb{Z} \Delta$.

For any vertex $x$ of $\Gamma$, we now define a cluster additive function $h_{x}$ as follows: Let $\mathcal{S}$ be any section containing $x$, let $h_{x}(x)=-1$ and $h_{x}(y)=0$ for $y \neq x$ in $\mathcal{S}$.

According to Theorem 3, we know that $h_{x}$ extends in a unique way to a cluster additive function $h_{x}$ on $\Gamma$ and this extension is independent of the choice of $\mathcal{S}$. We call $h_{x}$ the cluster hammock function for the vertex $x$.

Proof of the independency There is a section $\mathcal{S}^{\prime}$ with $x$ the unique sink of $\mathcal{S}^{\prime}$ and a section $\mathcal{S}^{\prime \prime}$ with $x$ the unique source of $\mathcal{S}^{\prime \prime}$, and all other sections containing $x$ are obtained from $\mathcal{S}^{\prime}$ or also $\mathcal{S}^{\prime \prime}$ by reflections at sinks or sources different from $x$. The corresponding cluster reflections $\sigma_{y}$ do not change the value 0 .

Note that the proof shows that $h_{x}(y)=0$ for all vertices $y \neq x$ which belong to the convex hull of $\mathcal{S}^{\prime}$ and $\mathcal{S}^{\prime \prime}$.

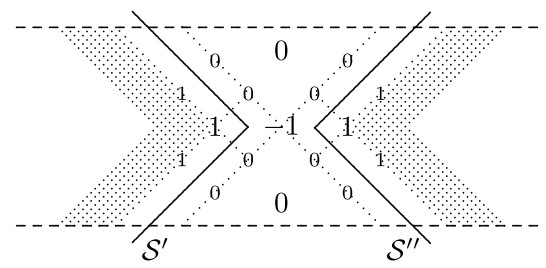

From now on, we will assume that $\Delta$ is a Dynkin quiver (if not stated otherwise).

Lemma Let $\Gamma=\mathbb{Z} \Delta$ with $\Delta$ a Dynkin quiver, then $h_{x}$ is $F$-invariant. The support of $h_{x}$ consists of

- the F-orbit of $x$ and $h_{x}$ takes the value -1 on these vertices, as well as

- the $F$-orbits of the hammock $H_{\tau^{-1} x_{x}}$ and here $h_{x}$ takes positive values, namely

$$
h_{x}(y)=h_{\tau^{-1} x}^{\prime}(y) \quad \text { for } y \in H_{\tau^{-1} x} \text {. }
$$




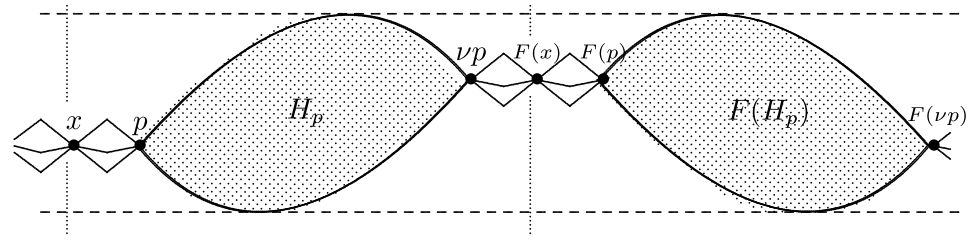

Here is a schematic illustration, where we write $p=\tau^{-1} x$ (the vertical dotted lines mark a fundamental domain for the action of $F$ ):

We have mentioned that $h_{x}$ is $F$-invariant: $h_{x}(y)=h_{x}(F y)$ for all $y \in \Gamma$. But this means also that $h_{x}=h_{F x}$. Thus, when dealing with a set of cluster hammock functions, we may restrict to look at those indexed by elements in some fixed fundamental domain for $F$.

Let us mention a property of the hammock functions $h_{p}^{\prime}$ (and of $h_{\tau p}$ ) which will be used in the next section. If there is a sectional path from $p$ to a vertex $y$, then $h_{p}^{\prime}(y) \geq 1$ (or better: in this case, $h_{p}^{\prime}(y)$ is the number of sectional paths from $p$ to $y$ ).

We call a subset $\mathcal{T}$ of $\mathbb{Z} \Delta$ confined provided there is a section $\mathcal{S}$ such that $\mathcal{T}$ is contained in the convex hull of $\mathcal{S}$ and $\tau \mathcal{S}$ [1]; note that this is the Auslander-Reiten quiver $\Gamma(A)$ of a hereditary algebra $A$ of type $\Delta$, with $\mathcal{S}$ the indecomposable projective $A$-modules, and $\tau \mathcal{S}[1]$ the indecomposable injective $A$-modules.

We call a subset $\mathcal{T}$ of $\Gamma$ a tilting set provided we can identify $\Gamma$ as a translation quiver with $D^{b}(\bmod A)$ for some hereditary algebra $A$ such that $\mathcal{T}$ are just the positions of the indecomposable direct summands of a tilting $A$-module. Subsets of tilting sets are called partial tilting sets.

Lemma Let $X, Y$ be non-isomorphic indecomposable A-modules. Then $\operatorname{Ext}^{1}(X, Y)$ $=0$ if and only if $h_{[Y]}([X])=0$.

Proof There is the Auslander-Reiten formula $\operatorname{Ext}^{1}(X, Y) \simeq D \operatorname{Hom}\left(\tau^{-} Y, X\right)$ and $\operatorname{dim} \operatorname{Hom}\left(\tau^{-} Y, X\right)=h_{\left[\tau^{-1} Y\right]}^{\prime}([X])=h_{[Y]}([X])$.

Corollary $A$ subset $\mathcal{T}$ of $\Gamma$ is partial tilting if and only if $\mathcal{T}$ is confined and $h_{x}\left(x^{\prime}\right)=$ 0 for all pairs $x \neq x^{\prime}$ in $\mathcal{T}$.

\section{Non-negative linear combinations of cluster hammock functions}

Again, we deal with a translation quiver $\Gamma=\mathbb{Z} \Delta$, where $\Delta$ is a Dynkin quiver, say with $n$ vertices.

Proposition 1 Consider a set $h_{1}, \ldots, h_{n}$ of cluster hammock functions. These functions are pairwise compatible if and only if there is a tilting set $\mathcal{T}$ such that any $h_{i}$ is of the form $h_{x}$ with $x \in \mathcal{T}$. 
Proof Let $\mathcal{T}$ be a tilting set and $x, x^{\prime} \in \mathcal{T}$. We have to show that $h_{x}, h_{x^{\prime}}$ are compatible. This is clear if $h_{x}=h_{x^{\prime}}$. Thus assume that $h_{x} \neq h_{x^{\prime}}$, thus $x$ and $x^{\prime}$ do not belong to the same $F$-orbit of $\Gamma_{0}$. Let $h_{x^{\prime}}(y)<0$, then $y$ belongs to the $F$-orbit of $x^{\prime}$, thus $h_{x}(y)=h_{x}\left(x^{\prime}\right)=0$. This shows that $h_{x}(y) h_{x^{\prime}}(y)=0$. Similarly, we see: if $h_{x}(y)<0$, then $h_{x}(y) h_{x^{\prime}}(y)=0$. For the remaining vertices $y$ we have both $h_{x}(y) \geq 0$ and $h_{x^{\prime}}(y) \geq 0$, thus also $h_{x}(y) h_{x^{\prime}}(y) \geq 0$.

Conversely, assume that the functions $h_{1}, \ldots, h_{n}$ are pairwise compatible. First, we show that for $h_{i} \neq h_{j}$, and $h_{j}=h_{y}$ for some vertex $y$, then $h_{i}(y)=0$. Namely, $h_{i}(y) h_{y}(y)=h_{i}(y) h_{j}(y) \geq 0$, and $h_{y}(y)=-1$ shows that $h_{i}(y) \leq 0$. But $h_{i}(y)<0$ would imply that $h_{i}=h_{y}$, a contradiction. Thus $h_{i}(y)=0$.

Now, let $h_{1}=h_{x}$ for some $x \in \Gamma_{0}$. Let $\mathcal{S}$ be the section in $\Gamma$ such that $\tau^{-2} F^{-1} x$ is the unique source. Let $\mathcal{S}^{\prime}=\mathcal{S}[1]$, this is the section with unique source $\tau^{-1} x$. Clearly, the convex hull $\mathcal{F}$ of $\mathcal{S}$ and $\mathcal{S}^{\prime}$ is a fundamental domain for $F$, thus $h_{i}=h_{x_{i}}$ for some $x_{i} \in \mathcal{F}$. Since $\tau^{-1} x$ is the unique source of $\mathcal{S}^{\prime}$, we see that $h_{x}(z)>0$ for all $z \in \mathcal{S}^{\prime}$. Assume that some $x_{j}$ belongs to $\mathcal{S}^{\prime}$, then $x \neq x_{j}$, thus $h_{x} \neq h_{x_{j}}$ (since $x, x_{j}$ belong to the fundamental domain $\mathcal{F}$ of $F$ ), but then we know that $h_{x}\left(x_{j}\right)=0$, a contradiction. In this way, we see that all the vertices $x_{i}$ belong to the convex hull of $\mathcal{S}$ and $\tau \mathcal{S}^{\prime}=\tau \mathcal{S}[1]$, thus the set $\mathcal{T}=\left\{x_{1}, \ldots, x_{n}\right\}$ is confined. Since also $h_{x}\left(x^{\prime}\right)=0$ for $x \neq x^{\prime}$ in $\mathcal{T}$, we see that $\mathcal{T}$ is a tilting set.

Corollary A linear combination $h=\sum_{x \in \mathcal{T}} n_{x} h_{x}$ with positive integers $n_{x}$ is cluster additive if and only if $\mathcal{T}$ is a partial tilting set.

Proof This is a direct consequence of Theorem 1 and Proposition 1.

Proposition 2 Let $f=\sum_{x \in \mathcal{T}} n_{x} h_{x}$ for some tilting set $\mathcal{T}$ and $n_{x} \in \mathbb{N}_{0}$, then $f(x)=$ $-n_{x}$ for $x \in \mathcal{T}$ and $f(y) \geq 0$ provided the intersection of $\mathcal{T}$ and the $F$-orbit of $y$ is empty. Thus

$$
f=\sum_{x \in \mathcal{T}} n_{x} h_{x}=-\sum_{x \in \mathcal{T}} f(x) h_{x}=\sum_{x \in \mathcal{T}} f(x)^{-} h_{x}=\sum_{x \in \Gamma^{0}} f(x)^{-} h_{x},
$$

where $\Gamma^{0}$ is the convex hull of some section $\mathcal{S}$ and $\tau \mathcal{S}[1]$.

Conjecture Let $\Gamma=\mathbb{Z} \Delta$ where $\Delta$ is a Dynkin quiver and let $f$ be cluster additive on $\Gamma$. Then $f$ is a non-negative linear combination of cluster hammock functions (and therefore of the form

$$
\sum_{x \in \mathcal{T}} n_{x} h_{x}
$$

for a tilting set $\mathcal{T}$ and integers $n_{x} \in \mathbb{N}_{0}$, for all $x \in \mathcal{T}$ ).

If this conjecture is true, then any cluster additive function satisfies the following properties:

(a) $f$ is $F$-invariant.

(b) $\left\{x \in \Gamma_{0} \mid f(x)<0\right\}$ is the union of the $F$-orbits of a partial tilting set. 
(c) There is a partial tilting set $\mathcal{T}$ with

$$
f=\sum_{x \in \mathcal{T}} f(x)^{-} h_{x} .
$$

A proof of the conjecture in the case $\mathbb{A}_{n}$ will be given in Sect. 9. We also note that it is not difficult to exhibit explicitely all the cluster additive functions on $\mathbb{Z} \Delta$, where $\Delta$ is a quiver of type $\mathbb{D}_{4}$, thus verifying the conjecture also in this case.

\section{The rectangle rule}

Lemma Let $f$ be cluster additive on the following translation quiver with $s \geq 1$, $t \geq 1$ :

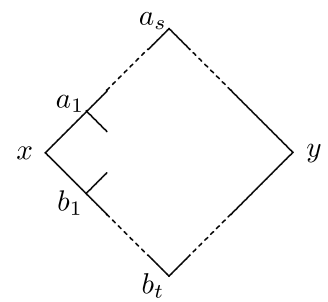

Then for $y=y(s, t)$ with $f(x) \leq 0$, we have

$$
f(y)=f(x)^{-}+\sum_{1 \leq i \leq s} f\left(a_{i}\right)^{-}+f\left(a_{s}\right)^{+}+\sum_{1 \leq j \leq t-1} f\left(b_{j}\right)^{-}+f\left(b_{t}\right)^{+} .
$$

In particular, $f(y) \geq f(x)^{-} \geq 0$.

Proof By induction on $s$ and $t$.

If $s=t=1$, then $f(y)=f\left(a_{1}\right)^{+}+f\left(b_{1}\right)^{+}-f(x)$.

Now assume that we know the formula for some $s, t$. Let us increase $s$ by 1 , thus we deal with

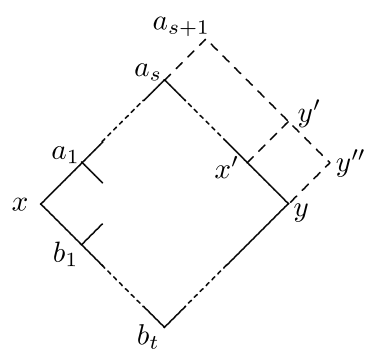

For $t=1$, we have $x^{\prime}=a_{s}$ and $y^{\prime}=a_{s+1}$, otherwise $x^{\prime}=y(s, t-1)$ and $y^{\prime}=$ $y(s+1, t-1)$. 
Now, consider first the case $t=1$. Then (since $f(y) \geq 0$ ):

$$
\begin{aligned}
f\left(y^{\prime \prime}\right)= & f\left(y^{\prime}\right)^{+}+f(y)-f\left(x^{\prime}\right) \\
= & f\left(a_{s+1}\right)^{+}+f(x)^{-}+\sum_{1 \leq i \leq s-1} f\left(a_{i}\right)^{-}+f\left(a_{s}\right)^{+} \\
& +\sum_{1 \leq j \leq t-1} f\left(b_{j}\right)^{-}+f\left(b_{t}\right)^{+}-f\left(a_{s}\right) \\
= & f\left(a_{s+1}\right)^{+}+f(x)^{-}+\sum_{1 \leq i \leq s} f\left(a_{i}\right)^{-}+\sum_{1 \leq j \leq t-1} f\left(b_{j}\right)^{-}+f\left(b_{t}\right)^{+}
\end{aligned}
$$

where the last equality comes from $f\left(a_{s}\right)^{+}-f\left(a_{s}\right)=f\left(a_{s}\right)^{-}$.

Second, let $t \geq 2$. Then both $f(y) \geq 0, f\left(y^{\prime}\right) \geq 0$, thus

$$
\begin{aligned}
f\left(y^{\prime \prime}\right)= & f\left(y^{\prime}\right)+f(y)-f\left(x^{\prime}\right) \\
= & f(x)^{-}+\sum_{1 \leq i \leq s} f\left(a_{i}\right)^{-}+f\left(a_{s+1}\right)^{+}+\sum_{1 \leq j \leq t-2} f\left(b_{j}\right)^{-}+f\left(b_{t-1}\right)^{+} \\
& +f(x)^{-}+\sum_{1 \leq i \leq s-1} f\left(a_{i}\right)^{-}+f\left(a_{s}\right)^{+}+\sum_{1 \leq j \leq t-1} f\left(b_{j}\right)^{-}+f\left(b_{t}\right)^{+} \\
& -f(x)^{-}-\sum_{1 \leq i \leq s-1} f\left(a_{i}\right)^{-}-f\left(a_{s}\right)^{+}-\sum_{1 \leq j \leq t-2} f\left(b_{j}\right)^{-}-f\left(b_{t-1}\right)^{+} \\
= & f(x)^{-}+\sum_{1 \leq i \leq s} f\left(a_{i}\right)^{-}+f\left(a_{s+1}\right)^{+}+\sum_{1 \leq j \leq t-1} f\left(b_{j}\right)^{-}+f\left(b_{t}\right)^{+},
\end{aligned}
$$

as we want.

By symmetry, the same argument works, if we increase $t$ instead of $s$. This completes the proof.

Extended version Let $f$ be cluster additive on the following translation quiver with $s \geq 1, t \geq 1$ :

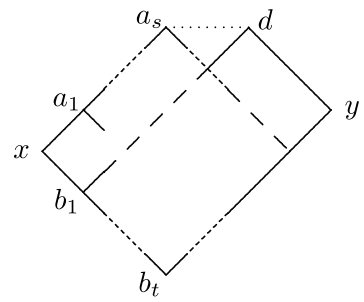

Then for $y=y(s+1, t)$ with $f(x) \leq 0$, we have

$$
f(y)=f(x)^{-}+\sum_{1 \leq i \leq s} f\left(a_{i}\right)^{-}+\sum_{1 \leq j \leq t-1} f\left(b_{j}\right)^{-}+f\left(b_{t}\right)^{+} .
$$


Proof We add a vertex $a_{s+1}$ and arrows $a_{s} \rightarrow a_{s+1}$ and $a_{s+1} \rightarrow d$, so that we obtain a rectangle. Also, we extend $f$ to be defined on the rectangle by setting $f\left(a_{s+1}\right)=0$. Then the extended function satisfies the cluster additivity condition on all the meshes of the rectangle and we can apply the lemma.

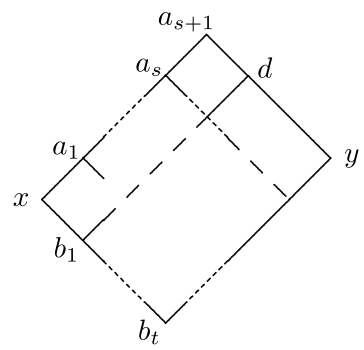

There is also a corresponding double extended version for dealing with $\mathbb{Z} \Delta$ where $\Delta$ is of type $\mathbb{A}_{s+t+1}$.

Double extended version Let $f$ be cluster additive on the following translation quiver with $s \geq 1, t \geq 1$ :

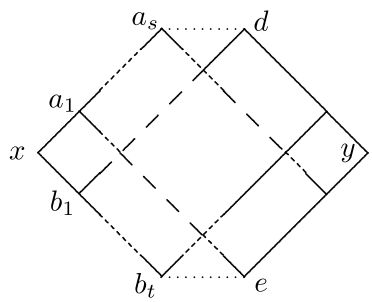

Then for $y=y(s+1, t+1)$ with $f(x) \leq 0$, we have

$$
f(y)=f(x)^{-}+\sum_{1 \leq i \leq s} f\left(a_{i}\right)^{-}+\sum_{1 \leq j \leq t} f\left(b_{j}\right)^{-} .
$$

\section{Wings}

Let $s \geq 0, t \geq 1$, let $y$ be a wing vertex of rank $s+t+1$, say with sectional paths

$$
p[1] \rightarrow p[2] \rightarrow \cdots \rightarrow p[s+t+1]=y, \quad y=[s+t+1] q \rightarrow \cdots \rightarrow[2] q \rightarrow[1] q .
$$

Lemma Assume that

$$
f(p[s]) \leq 0, \quad f(p[s+i]) \geq 0, \quad \text { for } 1 \leq i \leq t, \quad f(p[s+t+1]) \leq 0 .
$$

Then

$$
f([t] q)=-\min _{1 \leq i \leq t} f(p[s+i]) .
$$


Also, $f$ is non-negative on all vertices between $p[s+1]$ and $[1+t] q$ different from $y$.

Here is a sketch which exhibits the vertices in question in case $s \geq 1$ :

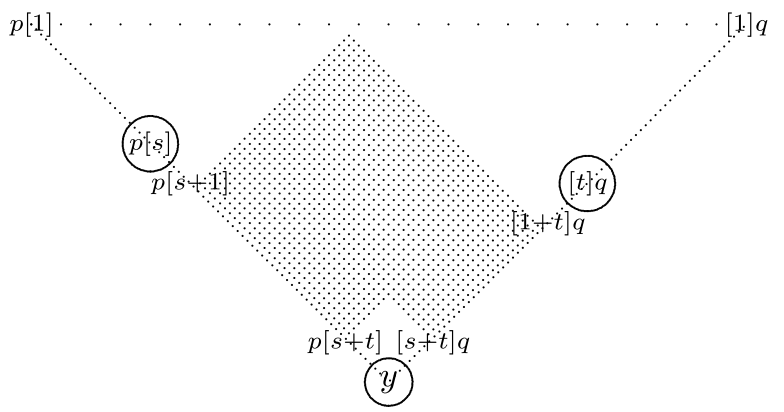

The case $s=0$ looks as follows:

$p]$ [1] $] q$

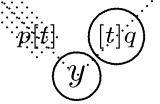

Proof Let us use the following labels for the relevant vertices of the wing:

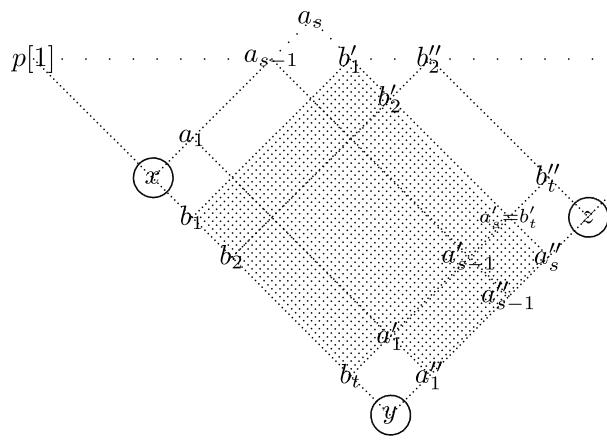

In particular

$$
x=p[s], \quad z=[t] q, \quad b_{i}=p[s+i], \quad a_{i}=\tau^{-i} p[s-i] .
$$


Note that we have added the vertex $a_{s}$ to the wing, (with additional arrows $a_{s-1} \rightarrow a_{s}$ and $\left.a_{s} \rightarrow b_{1}^{\prime}\right)$ and we put $f\left(a_{s}\right)=0$, as in the proof of the extended rectangle rule.

Using the new labels, the assumptions read:

$$
f(x) \leq 0, \quad f\left(b_{i}\right) \geq 0, \quad \text { for } 1 \leq i \leq t, \quad f(y) \leq 0
$$

and the assertion is that $f$ is non-negative on the shaded area (the vertices between $b_{1}$ and $a_{s}^{\prime \prime}$ different from $y$ ) and that

$$
f(z)=-\min \left(f\left(b_{i}\right) \mid 1 \leq i \leq t\right) .
$$

The rectangle rule asserts that $f$ is bounded below by $f(x)^{-}$on the rectangle between $\tau^{-1} x$ and $a_{s}^{\prime}=b_{t}^{\prime}$. By assumption, $f$ is non-negative on the vertices $b_{1}, \ldots, b_{t}$. Thus, concerning the non-negativity assertion, it remains to show that $f$ is nonnegative on the vertices $a_{1}^{\prime \prime}, \ldots, a_{s}^{\prime \prime}$.

The rectangle rule asserts that

$$
f\left(a_{i}^{\prime}\right)=f(x)^{-}+\sum_{j=1}^{i-1} f\left(a_{j}\right)^{-}+f\left(a_{i}\right)^{+}+\sum_{j=1}^{t-1} f\left(b_{j}\right)^{-}+f\left(b_{t}\right)^{+} .
$$

Since $f(y) \leq 0$ and $f\left(a_{1}^{\prime}\right) \geq 0$, we have $f\left(a_{1}^{\prime \prime}\right)=f\left(a_{1}^{\prime}\right)-f\left(b_{t}\right) \geq 0$. Assume by induction that we know that $f\left(a_{i}^{\prime \prime}\right)=f\left(a_{i}^{\prime}\right)-f\left(b_{t}\right) \geq 0$, then we get

$$
\begin{aligned}
f\left(a_{i+1}^{\prime \prime}\right)+f\left(a_{i}^{\prime}\right) & =f\left(a_{i}^{\prime \prime}\right)^{+}+f\left(a_{i+1}^{\prime}\right)^{+} \\
& =f\left(a_{i}^{\prime \prime}\right)+f\left(a_{i+1}^{\prime}\right) \\
& =f\left(a_{i}^{\prime}\right)-f\left(b_{t}\right)+f\left(a_{i+1}^{\prime}\right)
\end{aligned}
$$

and therefore

$$
f\left(a_{i+1}^{\prime \prime}\right)=f\left(a_{i+1}^{\prime}\right)-f\left(b_{t}\right) .
$$

By the rectangle rule for $a_{i+1}^{\prime}$ we see that $f\left(a_{i+1}^{\prime}\right)-f\left(b_{t}\right) \geq 0$ provided $i+1 \leq s$.

It remains to calculate the value $f(z)$.

Using induction on $i$, we show that

$$
f\left(b_{i}^{\prime \prime}\right)=f\left(b_{i}\right)-\min \left(f\left(b_{j}\right) \mid 1 \leq j<i\right)
$$

for $i \geq 2$.

The rectangle rule for $b_{i}^{\prime}$ yields

$$
\begin{aligned}
f\left(b_{i}^{\prime}\right) & =f(x)^{-}+\sum_{j=1}^{s-1} f\left(a_{j}\right)^{-}+f\left(a_{s}\right)^{+}+\sum_{j=1}^{i-1} f\left(b_{j}\right)^{-}+f\left(b_{i}\right)^{+} \\
& =f(x)^{-}+\sum_{j=1}^{s-1} f\left(a_{j}\right)^{-}+f\left(b_{i}\right),
\end{aligned}
$$


since $f\left(a_{s}\right)=0$ and all $f\left(b_{j}\right) \geq 0$. Similarly, for $b_{i+1}^{\prime}$ we get:

$$
f\left(b_{i+1}^{\prime}\right)=f(x)^{-}+\sum_{j=1}^{s-1} f\left(a_{j}\right)^{-}+f\left(b_{i+1}\right),
$$

thus

$$
f\left(b_{i+1}^{\prime}\right)-f\left(b_{i}^{\prime}\right)=f\left(b_{i+1}\right)-f\left(b_{i}\right) .
$$

For $i=2$, we have

$$
f\left(b_{2}^{\prime \prime}\right)=f\left(b_{2}^{\prime}\right)-f\left(b_{1}^{\prime}\right)
$$

since $f\left(b_{2}^{\prime}\right) \geq 0$, thus

$$
f\left(b_{2}^{\prime \prime}\right)=f\left(b_{2}^{\prime}\right)-f\left(b_{1}^{\prime}\right)=f\left(b_{2}\right)-f\left(b_{1}\right)=f\left(b_{2}\right)-\min \left(f\left(b_{j}\right) \mid 1 \leq j<2\right),
$$

as we have claimed.

Similarly, we have for all $i \geq 2$

$$
\begin{aligned}
f\left(b_{i+1}^{\prime \prime}\right) & =f\left(b_{i}^{\prime \prime}\right)^{+}+f\left(b_{i+1}^{\prime}\right)-f\left(b_{i}^{\prime}\right) \\
& =f\left(b_{i}^{\prime \prime}\right)^{+}+f\left(b_{i+1}\right)-f\left(b_{i}\right) .
\end{aligned}
$$

By induction, we may assume that

$$
f\left(b_{i}^{\prime \prime}\right)=f\left(b_{i}\right)-\min \left(f\left(b_{j}\right) \mid 1 \leq j<i\right),
$$

and we have to distinguish two cases:

First, assume that $f\left(b_{i}^{\prime \prime}\right) \leq 0$. Then $f\left(b_{i}^{\prime \prime}\right)^{+}=0$ and $f\left(b_{i}\right) \leq \min \left(f\left(b_{j}\right) \mid 1 \leq j<\right.$ $i)$, so that $\min \left(f\left(b_{j}\right) \mid 1 \leq j \leq i\right)=f\left(b_{i}\right)$. Thus

$$
\begin{aligned}
f\left(b_{i+1}^{\prime \prime}\right) & =f\left(b_{i}^{\prime \prime}\right)^{+}+f\left(b_{i+1}\right)-f\left(b_{i}\right) \\
& =0+f\left(b_{i+1}\right)-\min \left(f\left(b_{j}\right) \mid 1 \leq j \leq i\right),
\end{aligned}
$$

as we want to show.

In the second case, $f\left(b_{i}^{\prime \prime}\right) \geq 0$, thus $f\left(b_{i}^{\prime \prime}\right)^{+}=f\left(b_{i}^{\prime \prime}\right)$ and $f\left(b_{i}\right) \geq \min \left(f\left(b_{j}\right) \mid\right.$ $1 \leq j<i)$, so that $\min \left(f\left(b_{j}\right) \mid 1 \leq j \leq i\right)=\min \left(f\left(b_{j}\right) \mid 1 \leq j<i\right)$. Thus

$$
\begin{aligned}
f\left(b_{i+1}^{\prime \prime}\right) & =f\left(b_{i}^{\prime \prime}\right)^{+}+f\left(b_{i+1}\right)-f\left(b_{i}\right) \\
& =f\left(b_{i}\right)-\min \left(f\left(b_{j}\right) \mid 1 \leq j<i\right)+f\left(b_{i+1}\right)-f\left(b_{i}\right) \\
& =-\min \left(f\left(b_{j}\right) \mid 1 \leq j \leq i\right)+f\left(b_{i+1}\right) .
\end{aligned}
$$

Thus we see that

$$
f\left(b_{t}^{\prime \prime}\right)^{+}=f\left(b_{t}\right)-\min \left(f\left(b_{i}\right) \mid 1 \leq i \leq t\right) .
$$


On the other hand, the calculations in the first part of the proof had shown that $f\left(a_{s}^{\prime \prime}\right) \geq 0$ and that

$$
f\left(a_{s}^{\prime \prime}\right)-f\left(a_{s}^{\prime}\right)=-f\left(b_{t}\right)
$$

It follows that

$$
\begin{aligned}
f(z) & =f\left(b_{t}^{\prime \prime}\right)^{+}+f\left(a_{s}^{\prime \prime}\right)-f\left(a_{s}^{\prime}\right)=f\left(b_{t}^{\prime \prime}\right)^{+}+f\left(a_{s}^{\prime \prime}\right)^{+}-f\left(a_{s}^{\prime}\right) \\
& =f\left(b_{t}^{\prime \prime}\right)^{+}-f\left(b_{t}\right)=f\left(b_{t}\right)-\min \left(f\left(b_{i}\right) \mid 1 \leq i \leq t\right)-f\left(b_{t}\right) \\
& =-\min \left(f\left(b_{i}\right) \mid 1 \leq i \leq t\right) .
\end{aligned}
$$

This completes the proof.

\section{The case $\Gamma=\mathbb{Z} \mathbb{A}_{n}$}

Consider now the case $\Gamma=\mathbb{Z} \Delta$ with $\Delta$ of type $\mathbb{A}_{n}$.

Theorem 4 Let $\Gamma=\mathbb{Z} \Delta$ with $\Delta$ of type $\mathbb{A}_{n}$. Then any cluster additive function on $\Gamma$ is a non-negative linear combination of cluster hammock functions.

If $n=1$, then any cluster additive function on $\Gamma$ is a non-negative multiple of one of the two cluster hammock functions. Thus, we can assume that $n \geq 2$.

Let $f$ be a cluster additive function on $\Gamma$.

(1) If $z$ is a vertex of $\Gamma$ with $f(z) \leq 0$, then there is a vertex $z^{\prime} \neq z$ with $f\left(z^{\prime}\right) \leq 0$ and a sectional path from $z$ to $z^{\prime}$ or from $z^{\prime}$ to $z$.

Proof Since $n \geq 2$, there is an arrows $a_{1} \rightarrow a_{0}=z$. Choose $m$ maximal such that there exists a sectional path

$$
a_{m} \rightarrow \cdots \rightarrow a_{1} \rightarrow a_{0}=z
$$

If $f\left(a_{i}\right) \leq 0$ for some $1 \leq i \leq m$, then let $z^{\prime}=a_{i}$. Otherwise we consider the wing with corners

$$
p[1]=a_{m}, \quad z, \quad[1] q=\tau^{-m} a_{m} .
$$

The wing lemma (with $s=0$ ) asserts that $f\left(z^{\prime}\right) \leq 0$ (even $f\left(z^{\prime}\right)<0$ ) for $z^{\prime}=$ $\tau^{-1} a_{1}$.

(2) If $f(z)<0$ for some vertex $z$, then $f(z)=f(F z)$ and

$$
f(z)^{-} h_{z} \leq f
$$

Proof According to (1), there is a vertex $y=z^{\prime}$ with $f(y) \leq 0$ and a sectional path from $z$ to $y$ or from $y$ to $z$. Up to duality, we can assume that there is a sectional path from $y$ to $z$ (otherwise we consider instead of $\Gamma$ the opposite translation quiver). 
Also, we can assume that we choose $y$ such that the path from $y$ to $z$ is of smallest possible length (thus $f$ is positive on all the vertices between $y$ and $z$ ). Consider the wing with corners

$$
p[1], \quad y, \quad[1] q,
$$

thus there are sectional paths

$$
p[1] \rightarrow p[2] \rightarrow \cdots \rightarrow p[m]=y, \quad y=[m] q \rightarrow \cdots \rightarrow[2] q \rightarrow[1] q
$$

and $z$ is one of the vertices $[i] q$ with $1 \leq j<m$. Let $s \geq 0$ be maximal with $f(p[s]) \leq$ 0 and $t=m-s-1$. We claim that $t \geq 1$ and that $z=[t] q$.

First of all, for $t=0$, the rectangle rule would imply that $f([j] q) \geq 0$ for $1 \leq j<$ $m$, but $z$ is of the form $[j] q$ and $f(z)<0$.

This means that we can use the wing lemma, it asserts that

$$
f([t] q)=-\min \{f(p[s+i]) \mid 1 \leq i \leq t\}
$$

and that $f([j] q) \geq 0$ for $1+t \leq j \leq s+t$. Since $f(z)<0$, with $z$ of the form $[j] q$ and $j \leq s+t$, it follows that $j \leq t$. On the other hand, we know that $f$ is positive on all vertices between $y$ and $z$, thus we see that $j=t$.

Let $x=p[s], b_{i}=p[s+i]$, and $z=[t] q$ and note that we have $f(x) \leq 0, f(y) \leq$ 0 , and $f\left(b_{i}\right) \geq 1$ for $1 \leq i \leq t$. This yields the upper wing in the following picture, namely the wing with corners

$$
p[1], \quad y, \quad[1] q .
$$

$p[1]$

$x$

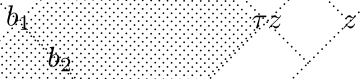

6

$y$

$F^{-1} z \tau^{-1} F^{-1}$

$-\cdots[n] q$

$p[n]$

According to the wing lemma, we know that

$$
f(z)=-\min \left(f\left(b_{i}\right) \mid 1 \leq i \leq t\right)<0 .
$$

But starting with $x$ and $y$, we may also look at the wing with corners

$$
[n] q, \quad x, \quad p[n],
$$


and use the dual argument: the dual of the wing lemma concerns the vertex $F^{-1} z$ (as well as the vertices between $F^{-1} z$ and $x$ ), it yields

$$
f\left(F^{-1} z\right)=-\min \left(f\left(b_{i}\right) \mid 1 \leq i \leq t\right) .
$$

This shows that

$$
f(z)=f\left(F^{-1} z\right) .
$$

Also, the rectangle rule for $F^{-1} z$ (or the dual rectangle rule for $z$ ) assert that $f$ is bounded from below by $f(z)^{-}=-f(z)$ on the rectangle starting with $\tau^{-1} F^{-1} z$ and ending with $\tau z$ (the shaded area).

Using induction as well as duality, we see that $f\left(F^{a} z\right)=f(z)$ for all $a \in \mathbb{Z}$. Also, it follows that

$$
f(z)^{-} h_{z} \leq f
$$

Proof of Theorem 4 Choose some section $\mathcal{S}$. Given a function $g$ on the set of vertices of $\Gamma$, we write

$$
|g|_{\mathcal{S}}=\sum_{x \in \mathcal{S}}|g(x)|
$$

thus $|g|_{\mathcal{S}}=0$ if and only if $g(x)=0$ for all $x \in \mathcal{S}$. In case $g$ is cluster additive, we know from Sect. 1 that $|g|_{\mathcal{S}}=0$ if and only if $g$ is the zero function.

We want to show any cluster additive function $f$ on $\Gamma$ is a non-negative linear combination of cluster hammock functions. We use induction on $|f| \mathcal{S}$. If $|f|_{\mathcal{S}}=0$, then $f$ is the zero function.

Now assume that $|f|_{\mathcal{S}}>0$. According to the assertion (5) in Sect. 1, there is some vertex $z$ with $f(z)<0$.

According to Theorem 2 and (2), we know that $h_{z} \leq f(z)^{-} h_{z} \leq f$. We see by Theorem 2 that $f-h_{z}$ is cluster additive again, and $\left|f-h_{z}\right|_{\mathcal{S}}<|f|_{\mathcal{S}}$. Thus, by induction, $f-h_{z}$ is a non-negative linear combination of cluster hammock functions and then also $f=\left(f-h_{z}\right)+h_{z}$ is a non-negative linear combination of cluster hammock functions. This completes the proof.

\section{Cluster-tilted algebras}

Let $A$ be a finite-dimensional hereditary $k$-algebra ( $k$ an algebraically closed field). Let $T$ be a tilting $A$-module, $\mathcal{T}$ the set of isomorphism classes of indecomposable direct summands of $T$, and $F \mathcal{T}$ the union of the $F$-orbits which contain elements of $\mathcal{T}$. Let $B$ be the opposite endomorphism ring of $T$ in the cluster category $\mathcal{C}=$ $D^{b}(\bmod A) / F($ see $[4])$, thus $B$ is a cluster tilted algebra.

Define a function $d_{T}$ on the Auslander-Reiten quiver $\Gamma$ of $D^{b}(\bmod A)$ as follows: Consider the projection

$$
D^{b}(\bmod A) \longrightarrow D^{b}(\bmod A) / F=\mathcal{C}_{A} \longrightarrow \mathcal{C}_{A} /\langle T\rangle=\bmod B,
$$

and denote it by $\pi$ (here, we use that we can identify $\mathcal{C}_{A} /\langle T\rangle$ with mod $B$ according to [5]). 
Let $y$ be a vertex of $\Gamma$, thus the isomorphism class of an indecomposable object in $D^{b}(\bmod A)$. If $y$ is not in $F \mathcal{T}$, then $\pi(y)$ is the isomorphism class of an indecomposable $B$-module and we denote by $d_{T}(y)$ its $k$-dimension. On the other hand, if the $F$-orbit of $y$ contains an element $x$ of $\mathcal{T}$, and $x=[X]$, where $X$ is an indecomposable direct summand of $T$, then let $d_{T}(y)=-n_{x}$, where $n_{x}$ is the Krull-Remak-Schmidt multiplicity of $X$ in $T$, note that this is also the $k$-dimension of the corresponding simple $B$-module $S_{x}$. In this way we obtain a function

$$
d_{T}: \Gamma_{0} \rightarrow \mathbb{Z}
$$

which obviously is $F$-invariant.

Of course, instead of looking at the $k$-dimension of the $B$-modules, one may also consider their length. In this way, one similarly defines the function

$$
l_{T}: \Gamma_{0} \rightarrow \mathbb{Z}
$$

with $l_{T}(y)$ the length of $\pi(y)$ in case $y$ is not in $F \mathcal{T}$, and with $l_{T}(y)=-1$ otherwise. If the tilting module $T$ is multiplicity free, then $l_{T}=d_{T}$. For a general tilting module $T$, let $T^{\prime}$ be multiplicity free with the same indecomposable direct summands as $T$, then $l_{T}=d_{T^{\prime}}$.

Let us assume now that $A$ is the path algebra of a Dynkin quiver.

Lemma The function $d_{T}$ on $\Gamma$ is cluster additive and we have

$$
d_{T}=\sum_{x \in \mathcal{T}} n_{x} h_{x}
$$

Proof We use the projection $\pi$ of $\Gamma=\mathbb{Z} \Delta$ onto the Auslander-Reiten quiver of $B$ as defined above, in particular the identification of $\mathcal{C}_{A} /\langle T\rangle$ with $\bmod B$ given by [5].

Let us consider the mesh of $\mathbb{Z} \Delta$ ending in $z$, say with arrows $y_{i} \rightarrow z, 1 \leq i \leq s$. We assume that the vertices $y_{r+1}, \ldots, y_{s}$ belong to $F \mathcal{T}$, and $y_{1}, \ldots, y_{r}$ not.

First, consider the case that neither $z$ nor $\tau z$ belong to $F \mathcal{T}$, thus we may consider the Auslander-Reiten sequence ending in $Z$. By the assumption on the $y_{i}$, we see that the Auslander-Reiten sequence has the form

$$
0 \rightarrow \tau Z \rightarrow \bigoplus_{i=1}^{r} Y_{i}^{m_{i}} \rightarrow Z \rightarrow 0,
$$

with indecomposable $B$-modules $Z$ and $Y_{i}$ such that $[Z]=z,\left[Y_{i}\right]=y_{i}$ and where $m_{i}=m_{y_{i}, z}$. It follows that

$$
\begin{aligned}
d_{T}(z)+d_{T}(\tau z) & =\operatorname{dim} Z+\operatorname{dim} \tau Z \\
& =\sum_{i=1}^{r} m_{i} \operatorname{dim} Y_{i}=\sum_{i=1}^{r} m_{y_{i}, z} d_{T}\left(y_{i}\right) \\
& =\sum_{i=1}^{s} m_{y_{i}, z} d_{T}\left(y_{i}\right)^{+}
\end{aligned}
$$


since $d_{T}\left(y_{i}\right)<0$ for $r+1 \leq i \leq s$.

Next, let $\tau z$ belong to $F \mathcal{T}$, thus $z$ is a projective vertex, say $z=[Z]$ for some indecomposable projective $B$-module $Z$. By the assumption on the $y_{i}$, the radical of $Z$ has the form $\operatorname{rad} Z=\bigoplus_{i=1}^{r} Y_{i}^{m_{i}}$ and $Z / \operatorname{rad} Z$ has dimension $n_{\tau z}$. This means

$$
\begin{aligned}
d_{T}(z)+d_{T}(\tau z) & =\operatorname{dim} Z-\operatorname{dim} Z / \operatorname{rad} Z=\operatorname{dim} \operatorname{rad} Z \\
& =\sum_{i=1}^{r} m_{i} \operatorname{dim} Y_{i}=\sum_{i=1}^{r} m_{y_{i}, z} d_{T}\left(y_{i}\right) \\
& =\sum_{i=1}^{s} m_{y_{i}, z} d_{T}\left(y_{i}\right)^{+}
\end{aligned}
$$

Finally, we have to consider the case where $z$ belongs to $F \mathcal{T}$. This case is dual to the previous one, now $\tau z=[X]$ for some indecomposable injective $B$-module $X$ and the socle of $X$ has dimension $n_{z}$.

The Jordan-Hölder theorem for $\bmod B$ shows that $d_{T}$ is just the sum of the various functions $n_{x} h_{x}$ with $x \in \mathcal{T}$; namely, if $y$ is a vertex of $\Gamma$, such that $\pi(y)$ is the isomorphism class of an indecomposable $B$-module $N$, then $h_{x}(y)$ is just the Jordan-Hölder multiplicity of the simple $B$-module $S_{x}$ in $N$.

We can use the cluster algebras in order to prove our conjecture for an $F$-invariant cluster additive function $f$ provided two conditions on the position of the vertices $x$ with $f(x) \leq 0$ are satisfied.

Proposition Let $f$ be a cluster additive function on $\Gamma=\mathbb{Z} \Delta$ with $\Delta$ a Dynkin quiver. Assume that $f$ is $F$-invariant and that there is a tilting set $\mathcal{T}$ with the following two properties:

(a) If $x$ belongs to $\mathcal{T}$, then $f(x) \leq 0$.

(b) If $f(x)<0$, then $x$ belongs to the $F$-orbit of an element of $\mathcal{T}$.

Then $f$ is a non-negative linear combination of cluster hammock functions.

Proof We identify $\Gamma=\mathbb{Z} \Delta$ with the Auslander-Reiten quiver of $D^{b}(\bmod A)$ where $A$ is a finite-dimensional hereditary algebra and where $T$ is a tilting $A$-module such that $\mathcal{T}$ is the set of isomorphism classes of indecomposable direct summands of $T$. Let $B$ be the opposite endomorphism $\operatorname{ring}$ of $T$ in $\mathcal{C}_{A}=D^{b}(\bmod A) / F$. We form the factor category $D^{b}(\bmod A) /\left\langle F^{i} T \mid i \in \mathbb{Z}\right\rangle$, this is the module category of a Galois cover $\widetilde{B}$ of $B$ (with Galois group $\mathbb{Z}$ ). Thus, the Auslander-Reiten quiver $\Gamma^{\prime}=\Gamma(\widetilde{B})$ of mod $\widetilde{B}$ is the translation subquiver obtained from $\Gamma$ by deleting the $F$-orbits of the vertices in $\mathcal{T}$.

Denote by $f^{\prime}$ the restriction of $f$ to $\Gamma^{\prime}$. By assumption (b), $f^{\prime}$ takes values in $\mathbb{N}_{0}$, is cluster additive, thus additive on $\Gamma(\widetilde{B})$ and $F$-invariant; thus it induces an additive function $f^{\prime \prime}$ on $\Gamma^{\prime \prime}=\Gamma(B)=\Gamma(\widetilde{B}) / F=\Gamma^{\prime} / F$ with values in $\mathbb{N}_{0}$. According to Butler [6], $f^{\prime \prime}$ is additive on all exact sequences, thus it is a linear combination of the "hammock functions" $h_{p}^{\prime \prime}$ for $\bmod B$, where $p$ runs through the set of indecomposable projective $B$-modules. If we compose these functions $h_{p}^{\prime \prime}$ with the projection $\Gamma^{\prime} \rightarrow$ 
$\Gamma^{\prime} / F=\Gamma^{\prime \prime}$, we obtain just the restriction of $h_{p}^{\prime}$ to $\Gamma^{\prime}$, where $p=\tau^{-1} x$ for some $x \in \mathcal{T}$. Thus, there are integers $n_{p}$ such that

$$
f^{\prime \prime}=\sum_{p} n_{p} h_{p}^{\prime \prime},
$$

and therefore

$$
f\left|\Gamma^{\prime}=f^{\prime}=\sum_{p} n_{p} h_{p}^{\prime}\right| \Gamma^{\prime}
$$

If $P^{\prime}$ is an indecomposable projective $B$-module with isomorphism class $p^{\prime}$ and $S^{\prime}$ is its top (a simple $B$-module), then

$$
n_{p^{\prime}}=\sum_{p} n_{p} h_{p}^{\prime \prime}\left(\left[S^{\prime}\right]\right)=f^{\prime \prime}\left(\left[S^{\prime}\right]\right) \geq 0
$$

(here we use that $f^{\prime}$ takes values in $\mathbb{N}_{0}$ ), thus all the coefficients $n_{p}$ are non-negative.

We have seen that $f$ and $h=\sum_{p} n_{p} h_{p}^{\prime}$ coincide on $\Gamma^{\prime}$, it remains to show that they also coincide on $\mathcal{T}$. Let $x \in \mathcal{T}$, thus $p=\tau^{-1} x$ is in $\Gamma_{0}^{\prime}$ and

$$
\begin{aligned}
f(x) & =-f(p)+\sum_{y \in \Gamma_{0}} m_{y, p} f(y)^{+} \\
& =-f(p)+\sum_{y \in \Gamma_{0}^{\prime}} m_{y, p(s)} f(y)^{+} \\
& =-h(p)+\sum_{y \in \Gamma_{0}^{\prime}} m_{y, p(s)} h(y)^{+} \\
& =-h(p)+\sum_{y \in \Gamma_{0}} m_{y, p(s)} h(y)^{+} \\
& =h(x),
\end{aligned}
$$

where we have used that both $f$ and $h$ are cluster additive, that they coincide on $\Gamma^{\prime}$ and have positive values only on vertices in $\Gamma_{0}^{\prime}$ (condition (a)). This completes the proof that $f=h$.

If a cluster additive function on $\Gamma$ is a non-negative linear combination of cluster hammock functions, then also the following properties are satisfied:

(d) $f=d_{T}$ for some partial tilting module $T$.

(e) If $f$ takes values in $\{-1\} \cup \mathbb{N}_{0}$, then $f=d_{T}$ for some multiplicity-free partial tilting module $T$, if $f$ takes values in $\mathbb{Z} \backslash\{0\}$, then $f=d_{T}$ for some tilting module $T$.

We end this section by giving an interpretation of the exchange property of cluster tilting objects in a cluster category in terms of the cluster hammock functions. Thus, suppose that we deal with a tilting set $\mathcal{T}$ in $\mathbb{Z} \Delta$, where $\Delta$ is a Dynkin quiver. Let us 
look at the hammock $h_{x}$ for some $x \in \mathcal{T}$. Let $\mathcal{T}^{\prime}=\mathcal{T} \backslash\{x\}$. We claim that there are precisely two $F$-orbits of vertices of $\Gamma$ which are not in the support of any function $h_{y}$ with $y \in \mathcal{T}^{\prime}$. Of course, one of these vertices is $x$ itself, since $h_{y}(x)=0$ for all $y \in \mathcal{T}^{\prime}$. In order to find the other orbit, we only have to consider the vertices $z$ which do not belong to $F \mathcal{T}$. As above, we know that $\pi(z)$ is the isomorphism class $[N]$ of an indecomposable $B$-module, say $N$. Now either $[N]=\left[S_{x}\right]$, then indeed $h_{y}(z)=0$ for all $y \in \mathcal{T}^{\prime}$ (since $N$ has no composition factor of the form $S_{y}$ ), or else $N$ is not isomorphic to $S_{x}$, but then $N$ has at least one composition different from $S_{x}$, say $S_{y}$ with $y \in \mathcal{T}^{\prime}$, and therefore $h_{y}(z) \neq 0$. This shows that the second orbit consists of the vertices $z$ such that $\pi(z)=\left[S_{x}\right]$. (But a warning is necessary: the position of $z$ with $\pi(z)=\left[S_{x}\right]$ in the support of $h_{x}$ does not only depend on $h_{x}$ itself, as already the case $\mathbb{A}_{2}$ shows.)

\section{Final remarks}

1. The main results and conjectures of this note concern the translation quivers $\mathbb{Z} \Delta$ with $\Delta$ a quiver whose underlying graph is a simply laced Dynkin diagram. But there is no problem to extend the considerations to the case of an arbitrary (not necessarily simply laced) Dynkin diagram (thus dealing with the cases $\mathbb{B}_{n}, \mathbb{C}_{n}, \mathbb{F}_{4}, \mathbb{G}_{2}$ ). In order to do so, we need the notion of a valued translation quiver.

A valued translation quiver $\Gamma=\left(\Gamma_{0}, \Gamma_{1}, \tau, m, m^{\prime}\right)$ is given by a translation quiver $\left(\Gamma_{0}, \Gamma_{1}, \tau\right)$ with the property that there is at most one arrow $x \rightarrow y$ for any pair $x, y$ of vertices and two functions $m, m^{\prime}: \Gamma_{1} \rightarrow \mathbb{N}_{1}$ such that

$$
m(\tau z, y)=m^{\prime}(y, z) \text { and } \quad m^{\prime}(\tau z, y)=m(y, z),
$$

for any arrow $y \rightarrow z$ in $\Gamma$ with $z$ a non-projective vertex. In case $m=m^{\prime}$, then we may consider $\left(\Gamma_{0}, \Gamma_{1}, \tau, m, m^{\prime}\right)$ as an ordinary translation quiver by replacing any arrow $x \rightarrow y$ by $m(x, y)$ arrows.

For example, the valued translation quiver $\mathbb{Z B}_{3}$ has the following form (in such pictures it is sufficient to add the pair of numbers $\left(m(x, y), m^{\prime}(x, y)\right)$ to an arrow $x \rightarrow y$ only in case at least one of the numbers is greater than 1$)$ :

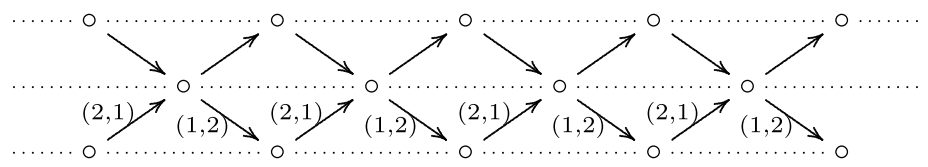

The valued translation quiver $\Gamma=\left(\Gamma_{0}, \Gamma_{1}, \tau, m, m^{\prime}\right)$ is said to be stable, if $\left(\Gamma_{0}, \Gamma_{1}, \tau\right)$ is stable.

Given a stable valued translation quiver $\Gamma$, a function $f: \Gamma_{0} \rightarrow \mathbb{Z}$ should be called cluster additive provided

$$
f(z)+f(\tau z)=\sum_{y \in \Gamma_{0}} m(y, z) f(y)^{+}, \quad \text { for all } z \in \Gamma_{0} .
$$


2. We should stress that cluster additive functions are definitely also of interest when dealing with stable translation quivers which are not related to translation quivers of the form $\mathbb{Z} \Delta$ with $\Delta$ a finite directed quiver. Examples of cluster additive functions on the translation quiver $\mathbb{Z} \mathbb{D}_{\infty}$ (as well as on $\mathbb{Z} \mathbb{A}_{\infty}^{\infty}$ ) have been exhibited in [12].

Acknowledgements The paper was written during a stay at the Hausdorff Research Institute for Mathematics, Bonn, January-April 2011, and is inspired by a number of lectures on the combinatorics of cluster categories. The author has to thank Guo Lingyan (Paris), but also to the referees for spotting misprints and inaccuracies in the first version of the paper.

\section{References}

1. Assem, I., Reutenauer, C., Smith, D.: Friezes. Adv. Math. 225, 3134-3165 (2010)

2. Bernstein, I.N., Gelfand, I.M., Ponomarev, V.A.: Coxeter functors and Gabriel's theorem. Usp. Mat. Nauk 28, 19-33 (1973). Russ. Math. Surv. 29, 17-32 (1973)

3. Brenner, S.: A combinatorial characterisation of finite Auslander-Reiten quivers. In: Dlab, V., Gabriel, P., Michler, G. (eds.) Representation Theory I. Finite Dimensional Algebras. LNM, vol. 1177, pp. 1349. Springer, Berlin (1986)

4. Buan, A.B., Marsh, R.J., Reineke, M., Reiten, I., Todorov, G.: Tilting theory and cluster combinatorics. Adv. Math. 204, 572-618 (2006)

5. Buan, A.B., Marsh, R.J., Reiten, I.: Cluster-tilted algebras. Trans. Am. Math. Soc. 359, 323-332 (2007)

6. Butler, M.C.R.: Grothendieck groups and almost split sequences. In: Roggenkamp, K.W. (ed.) Integral Representations and Applications. LNM, vol. 882, pp. 357-368. Springer, Berlin (1981)

7. Calderon, P., Chapoton, F., Schiffler, R.: Quivers with relations arising from clusters $\left(A_{n}\right.$-case). Trans. Am. Math. Soc. 358, 1347-1364 (2006)

8. Fomin, S., Zelevinsky, A.: Cluster algebras. I. Foundation. J. Am. Math. Soc. 15, 497-529 (2002)

9. Gabriel, P.: Auslander-Reiten sequences and representation-finite algebras. In: Representation Theory I. LNM, vol. 831, pp. 1-71. Springer, Berlin (1980)

10. Happel, D.: Triangulated Categories in the Representation Theory of Finite-Dimensional Algebras. LMS Lecture Note Series, vol. 119. Cambridge (1988)

11. Happel, D., Preiser, U., Ringel, C.M.: Vinberg's characterization of Dynkin diagrams using subadditive functions with application to DTr-periodic modules. In: Representation Theory II. LNM, vol. 832, pp. 280-294 (1980)

12. Ringel, C.M.: The minimal representation-infinite algebras which are special biserial. In: Skowroński, A., Yamagata, K. (eds.) Representations of Algebras and Related Topics, pp. 501-560. European Math. Soc. Publ. House, Zürich (2011)

13. Riedtmann, C.: Algebren, Darstellungsköcher, Überlagerungen und zurück. Comment. Math. Helv. 55, 199-224 (1980)

14. Ringel, C.M., Vossieck, D.: Hammocks. Proc. Lond. Math. Soc. 54(3), 216-246 (1987) 